\title{
NONLINEAR BREAKUP OF AN ELECTROHYDRODYNAMIC JET*
}

\author{
BY \\ S. K. MALIK AND M. SIINGH \\ Simon Fraser University
}

\begin{abstract}
A weakly nonlinear theory of the breakup of a jet held together with the capillary forces in the presence of an applied electric field is presented. It is shown that the jet breaks into the main drops and their satellites whose sizes are sensitive to the wavenumbers and the electric field. The satellites always exist when the electric field is applied to the jet which, however, is not the case when the electric field is absent.
\end{abstract}

1. Introduction. The stability of the capillary jet is one of the classical problems in fluid mechanics. The linear theory governing this phenomenon was given by Rayleigh [1] who also developed a mathematical model for the breakup of liquid jets into drops. Such a uniform model based on linear theory does not explain the experimental observations of Donnelly and Glaberson [2], and Rutland and Jameson [3]. These experiments indicate that the jet breaks up into a body of main drops interspersed with satellite drops whose size is a function of the wavenumber of the disturbance. These observations motivated Yuen [4] to formulate a nonlinear theory for the breakup of liquid jets which exhibits the existence of the satellites. This problem is of fundamental importance in a growing number of applications such as the production of lead shots, spray drying, electronic ink jet printing, fluid jet amplification and others in the synthetic and fertilizer industries.

The purpose of this paper is to investigate the nonlinear breakup of a laminar conducting liquid jet in the presence of an electric field. The theoretical as well as the experimental studies concerning the influence of electric fields on the surface waves at the capillary jets were investigated by Melcher [5]. This investigation is somewhat similar in physical characteristics to the nonlinear breakup of a self-gravitating column, considered earlier by Malik et al. [6]. The analysis of this study was confined within the framework of the linear theory. Recently, the satellite-drop formation has been recognized as a highly nonlinear behaviour. We present here a third order nonlinear theory pertaining to this problem by using the method of straining of the coordinates (see Nayfeh [7]).

The basic equations and the mathematical scheme for solving this nonlinear initial boundary value problem are given in Sec. 2. In Sec. 3 we recapitulate the linear theory. Sec. 4 and 5 are then devoted to obtaining the second and third order solutions of the nonlinear problem, respectively. It is shown that the perturbed surface of the jet assumes a nonsinusoidal shape resulting in secondary waves which is a direct consequence of the

\footnotetext{
* Received June 2, 1982.
} 
energy transfer between the harmonics of the various orders. In Sec. 6, we have calculated numercially the nonlinear breakup time by increasing the time variable in the perturbed surface equation until the amplitude of the surface wave attains the value of the radius of the undisturbed jet. This criteria of jet breakup successfully explains the experimental observations of Rutland and Jameson [3] as reported by Lafrance [8] who used Yuen's [4] theoretical solution to calculate the relative size of satellites and main drops as a function of the wavenumber. The fluid enclosed by the primary wave and the secondary wave is assumed to form the main and the satellite drop, respectively. The resultant bodies after the breakup are taken to be spherical in shape whose volumes we have derived by closed form integration. These volumes are then employed to obtain the radii of the main drops and their satellites.

2. Formulation of the problem. We consider an incompressible, inviscid fluid jet of radius $R_{0}$ held together by the capillary forces. The effects of the surrounding fluid are taken to be negligible. The jet is projected with a uniform speed $U_{0}$ along the axis of the cylinder. The cylinder is at a fixed potential $V_{0}$ relative to that of the jet, and its walls are far away from the surface of the jet. A radial electric field thus stresses the jet. The fluid is assumed to be highly conducted so as to exclude the contribution of the electric field from the fluid in the jet. Now, a periodic initial disturbance is given at the surface of the jet. The radius $b$ of the outer conducting cylinder is much larger than the perturbations wavelength of the jet. To describe the fluid motion, we use the moving frame of reference with the jet at rest. If $\left(r, x, t_{0}\right)$ is the coordinate system for the travelling jet and $(r, Z, t)$ for the jet at rest, the transformation connecting the two systems is given by

$$
Z=x-U_{0} t_{0}, t=x / U_{0} .
$$

If $\mathbf{V}(=\nabla \Omega)$ and $\mathbf{E}(=-\nabla \Phi)$ denote velocity field and the electric field, respectively, at any time $t$, then the equations governing the velocity potential $\Omega$ and the electric potential $\Phi$ are

$$
\begin{aligned}
& \nabla^{2} \Omega(r, \theta, Z)=0 \\
& \nabla^{2} \Phi(r, \theta, Z)=0
\end{aligned}
$$

for $r \leqslant R_{0}+\eta(Z, t)$, where $\eta(Z, t)$ denotes the elevation of the free surface measured from the unperturbed level. All the quantities are normalised with respect to the characteristic length $R_{0}$, the radius of the undisturbed jet, the characteristic speed $\left[T / \rho R_{0}\right]^{1 / 2}$, and the characteristic electric field parameter $R_{0} E_{0}^{2} / T$. Here, the density of the fluid is denoted by $\rho$, the surface tension by $T$, and the field strength at the surface of the undeformed jet by $E_{0}$. We shall employ C.G.S. units. For convenience, we introduce the following dimensionless variables, denoted by primes:

$$
\begin{aligned}
& Z=R_{0} Z^{\prime}, \quad r=R_{0} r^{\prime}, \quad t=\left(\rho R_{0} / T\right)^{1 / 2} R_{0} t^{\prime} \\
& \eta=R_{0} \eta^{\prime}, \quad b=R_{0} b^{\prime}, \quad \Omega=\left(T / \rho R_{0}\right)^{1 / 2} R_{0} \Omega^{\prime}, \\
& \Phi=\frac{V_{0}}{\ln \left(b / R_{0}\right)} \Phi^{\prime} .
\end{aligned}
$$


In the subsequent analysis, the primes on the dimensionless variables shall be dropped for brevity. The equations (1) and (2) are to be solved subject to the following boundary conditions:

(i) The kinematic condition that the fluid moves the interface is given by

$$
\begin{aligned}
& \frac{D}{D t}(r-\eta(Z, t)-1)=0 \quad \text { or } \\
& -\frac{\partial \eta}{\partial t}+\frac{\partial \Omega}{\partial r}-\frac{\partial \Omega}{\partial Z} \frac{\partial \eta}{\partial Z}=0 \quad \text { at } r=1+\eta(Z, t) .
\end{aligned}
$$

(ii) Since the fluid is conducting

$$
\begin{array}{ll}
\Phi=0 & \text { at } r=1+\eta(Z, t), \\
\Phi=1 & \text { at } Z=b .
\end{array}
$$

(iii) At the free surface, the normal stress is continuous (see Appendix A)

$$
\begin{aligned}
1-\frac{\partial \Omega}{\partial t}-\frac{1}{2}\left[\left(\frac{\partial \Omega}{\partial r}\right)^{2}+\left(\frac{\partial \Omega}{\partial Z}\right)^{2}\right]+\frac{\partial^{2} \eta}{\partial Z^{2}}\left[1+\left(\frac{\partial \eta}{\partial Z}\right)^{2}\right]^{-3 / 2} \\
-(1+\eta)^{-1}\left[1+\left(\frac{\partial \eta}{\partial Z}\right)^{2}\right]^{-1 / 2}+\frac{\alpha_{E}}{2}\left(\frac{\partial \Phi}{\partial \hat{n}}\right)^{2}=0 .
\end{aligned}
$$

where

$$
\alpha_{E}=\frac{R_{0} E_{0}^{2}}{4 \pi T}=\frac{V_{0}^{2}}{4 \pi T R_{0}},
$$

and where $\hat{n}$ denotes the unit normal drawn to the surface $r=1+\eta(Z, t)$. For a water jet of $0.1 \mathrm{~cm}$ radius, $\alpha_{E}$ is approximately 1 when the electric field strength is taken near the breakdown strength of a gas. For a water jet of radius $0.5 \mathrm{~cm}$, the corresponding value of $\alpha_{E}$ is 5. Melcher [5] used the values of $\alpha_{E}$ ranging from 0 to 2 in his experimental study of the water jet. At the time $t=0$, an axisymmetric disturbance of amplitude $\eta_{0}$ and wavenumber $k$ is imposed on the surface $r=1$ of the jet. The deformed surface now is given by

$$
r=R_{0}+\eta_{0} \cos (k Z),
$$

where the axis of the symmetry has been chosen as the $Z$-axis. By virtue of the conservation of mass,

$$
R_{0}=\left(1-\frac{1}{2} \eta_{0}^{2}\right)^{1 / 2} .
$$

We assume the initial conditions to be

$$
\begin{aligned}
\eta(Z, 0) & =\eta_{0} \cos (k Z)+\left(1-\frac{1}{2} \eta_{0}^{2}\right)^{1 / 2}-1, \\
\partial \eta(Z, 0) / \partial t & =0 .
\end{aligned}
$$

We wish to examine the weakly nonlinear stability problem posed by the equations (1)-(9). The method employed is that of the strained coordinates [7]. In order to describe the nonlinear interactions of small but finite amplitude waves, we write

$$
\Omega(r, Z, t)=\sum_{m=1}^{\infty} \eta_{0}^{m} \Omega_{m}(r, Z, t),
$$




$$
\Phi(r, Z, t)=\sum_{m=0}^{\infty} \eta_{0}^{m} \Phi_{m}(r, Z, t),
$$

and

$$
\eta(Z, t)=\sum_{m=1}^{\infty} \eta_{0}^{m} \eta_{m}(Z, t) .
$$

The above expansions are assumed to be uniformly valid for $-\infty<Z<\infty, 0 \leqslant t \leqslant T$, where $T$ is related to the breakup time for the growing perturbations, and is infinite for the stable oscillations of the surface.

We now introduce the strained coordinates:

$$
\begin{aligned}
& \tau=t \nu=t\left[\sum_{m=1}^{\infty} \eta_{0}^{m-1} \nu_{m}\right], \\
& \zeta=k_{c} Z=Z\left[\sum_{m=1}^{\infty} \eta_{0}^{m-1} k_{m}\right],
\end{aligned}
$$

where $\nu_{m}$ and $k_{m}$ are chosen so as to obtain the uniform valid solutions.

The boundary conditions (3), (4) and (5) are given at $r=1+\eta(Z, t)$. Since we do not have a priori information about the elevation $\eta(Z, t)$ of the free surface, we use Taylor's expansions of the various quantities appearing in the equations (3) to (5) about $r=1$ in powers of $\eta$, leading to the linear and the successive nonlinear partial differential equations of the various orders. The problem for any order can then be solved with the knowledge of the solutions of all the previous orders (see Nayfeh [9], Malik et al. [6]).

3. Linear theory. We substitute the expressions (10), (11) and (12) for $\Omega, \Phi$, and $\eta$, respectively into the field equations (1) and (2), the boundary conditions (3), (4) and (5) after having been reduced at $r=1$, and the intial conditions (8) and (9). We retain terms up to the first order in the small parameter $\eta_{0}$. Then, the first order problem $O\left(\eta_{0}\right)$ is characterized by

$$
\begin{aligned}
& L\left[\Omega_{1}\right]=\left(\frac{\partial^{2}}{\partial r^{2}}+\frac{1}{r} \frac{\partial}{\partial r}+k_{c}^{2} \frac{\partial^{2}}{\partial \xi^{2}}\right) \Omega_{1}=0, \\
& L\left[\Phi_{1}\right]=0 .
\end{aligned}
$$

The various boundary conditions at $r=1$ being

$$
\begin{gathered}
-\nu_{1} \frac{\partial \eta_{1}}{\partial \tau}+\frac{\partial \Omega_{1}}{\partial r}=0, \\
\Phi_{1}+\eta_{1} \frac{\partial \Phi_{0}}{\partial r}=0 \\
-\nu_{1} \frac{\partial \Omega_{1}}{\partial \tau}+\left(1+\frac{\partial^{2}}{\partial \xi^{2}}\right) \eta_{1}+\alpha_{E}\left[\frac{\partial \Phi_{1}}{\partial r} \frac{\partial \Phi_{0}}{\partial r}+\eta_{1} \frac{\partial \Phi_{0}}{\partial r} \cdot \frac{\partial^{2} \Phi_{0}}{\partial r^{2}}\right]=0,
\end{gathered}
$$

where

$$
\Phi_{0}=-\ln (r) .
$$


The initial conditions are

$$
\eta_{1}(\xi, 0)=\cos (K \xi), \quad \partial \eta_{1}(\xi, 0) / \partial \tau=0 .
$$

Here, the wave number $K$ in the strained coordinate system has the representation

$$
K=\frac{k}{\left(k_{1}+\varepsilon k_{2}+\varepsilon^{2} k_{3}+\cdots\right)} \text {. }
$$

For $\nu_{1}=1$, the solutions of equations (15)-(21) can be obtained as

$$
\begin{aligned}
\eta_{1}(\xi, \tau) & =\cosh \left(\omega_{1} \tau\right) \cos (K \xi), \\
\Omega_{1} & =\frac{\omega_{1}}{K} \frac{I_{0}(K r)}{I_{1}(K)} \sinh \left(\omega_{1} \tau\right) \cos (K \xi), \\
\Phi_{1} & =\frac{K_{0}(K r)}{K_{0}(K)} \cosh \left(\omega_{1} \tau\right) \cos (K \xi),
\end{aligned}
$$

where

$$
\begin{aligned}
& \omega_{1}^{2}=\frac{K}{I_{a}}\left(1-K^{2}\right)+\alpha_{E}(K \gamma(K)-1), \\
& I_{a}=\frac{I_{0}(K)}{I_{1}(K)}, \quad \gamma(K)=\frac{K_{1}(K)}{K_{0}(K)} .
\end{aligned}
$$

These solutions are stable for the deformations whose wavenumber is greater than $K_{\theta}$, where $K_{\theta}$ is given by the relation

$$
1-K^{2}+\alpha_{E}(K \gamma(K)-1)=0 .
$$

The value $K=K_{\theta}$ is the linear cutoff wavenumber which separates the stable from the unstable disturbances. The equation (25) reduces to Rayleigh's result for the long wavelength approximation. For $\alpha_{E}=0$, we recover the results of Lord Rayleigh [1] for the hydrodynamic jet whose maximum growth of instability is at $K=0.678$.

4. Second order solutions. The second order problem $O\left(\eta_{0}^{2}\right)$ is governed by

$$
\begin{aligned}
& L\left[\Omega_{2}\right]=-2 k_{1} k_{2}\left(\partial^{2} \Omega_{1} / \partial \xi^{2}\right), \\
& L\left[\Phi_{2}\right]=-2 k_{1} k_{2}\left(\partial^{2} \Phi_{1} / \partial \xi^{2}\right),
\end{aligned}
$$

with the boundary conditions at $r=1$ as

$$
\begin{aligned}
-\nu_{1} \frac{\partial \eta_{2}}{\partial \tau}+\frac{\partial \Omega_{2}}{\partial r} & =\nu_{2} \frac{\partial \eta_{1}}{\partial \tau}-\eta_{1} \frac{\partial^{2} \Omega_{1}}{\partial r^{2}}+\frac{\partial \Omega_{1}}{\partial \xi} \frac{\partial \eta_{1}}{\partial \xi}, \\
\Phi_{2}+\eta_{2} \frac{\partial \Phi_{0}}{\partial r} & =-\eta_{1} \frac{\partial \Phi_{1}}{\partial r}-\frac{\eta_{1}^{2}}{2} \frac{\partial^{2} \Phi_{0}}{\partial r^{2}}
\end{aligned}
$$




$$
\begin{aligned}
-\nu_{1} \frac{\partial \Omega_{2}}{\partial \tau}+ & \left(1+\frac{\partial^{2}}{\partial \xi^{2}}\right) \eta_{2}+\alpha_{E}\left[\left(\frac{\partial \Phi_{0}}{\partial r}\right)\left(\frac{\partial \Phi_{2}}{\partial r}\right)+\eta_{2}\left(\frac{\partial \Phi_{0}}{\partial r}\right)\left(\frac{\partial^{2} \Phi_{0}}{\partial r^{2}}\right)\right] \\
= & \nu_{2} \frac{\partial \Omega_{1}}{\partial \tau}+\eta_{1} \nu_{1}\left(\frac{\partial^{2} \Omega_{1}}{\partial \tau \partial r}\right)+\frac{1}{2}\left[\left(\frac{\partial \Omega_{1}}{\partial r}\right)^{2}+\left(\frac{\partial \Omega_{1}}{\partial \xi}\right)^{2}\right] \\
& -2 k_{2} \frac{\partial^{2} \eta_{1}}{\partial \xi^{2}}+\eta_{1}^{2}-\frac{1}{2}\left(\frac{\partial \eta_{1}}{\partial \xi}\right)^{2} \\
& -\frac{\alpha}{2}\left[\left(\frac{\partial \Phi_{1}}{\partial r}\right)^{2}+2 \eta_{1} \frac{\partial \Phi_{1}}{\partial r} \frac{\partial^{2} \Phi_{0}}{\partial r^{2}}\right. \\
& +2 \eta_{1}\left(\frac{\partial \Phi_{0}}{\partial r}\right)\left(\frac{\partial^{2} \Phi_{1}}{\partial r^{2}}\right)-2\left(\frac{\partial \Phi_{0}}{\partial r}\right)\left(\frac{\partial \eta_{1}}{\partial \xi}\right)\left(\frac{\partial \Phi_{1}}{\partial \xi}\right) \\
& \left.+\eta_{1}^{2}\left\{\left(\frac{\partial^{2} \Phi_{0}}{\partial r^{2}}\right)^{2}+\left(\frac{\partial \Phi_{0}}{\partial r}\right)\left(\frac{\partial^{3} \Phi_{0}}{\partial r^{3}}\right)\right\}-\left(\frac{\partial \Phi_{0}}{\partial r}\right)^{2}\left(\frac{\partial \eta_{1}}{\partial \xi}\right)^{2}\right],
\end{aligned}
$$

and the initial data

$$
\eta_{2}(\xi, 0)=-\frac{1}{4}, \quad \partial \eta_{2}(\xi, 0) / \partial \tau=0 .
$$

To obtain the solution from equations (28)-(33), we assume

$$
\eta_{2}(\xi, \tau)=B_{22}(\tau) \cos (2 K \xi)+D_{2}(\tau) .
$$

Substitution from equations (34), (22)-(26) into equations (28)-(33) yields the following uniformly valid second order solutions:

$$
\begin{aligned}
B_{22}(\tau)= & a_{22} \cosh \left(\omega_{2} \tau\right)+b_{22} \cosh \left(2 \omega_{1} \tau\right)+c_{22}, \\
\Phi_{2}(\tau)= & \Phi_{22}(\tau) \frac{K_{0}(2 K r)}{K_{0}(2 K)} \cos (2 K \xi) \\
\Omega_{2}(\tau)= & {\left[\left(\frac{\partial}{\partial \tau} B_{22}(\tau)\right) \frac{I_{0}(2 K r)}{2 K I_{1}(2 K)}+\frac{P_{22}(\tau)}{2 K} \frac{I_{0}(2 K r)}{I_{1}(2 K)} \sinh \left(2 \omega_{1} \tau\right)\right] \cos (2 K \xi) } \\
& +F(\tau),
\end{aligned}
$$

where

$$
\begin{aligned}
a_{22} & =\left(b_{22}+c_{22}\right) \\
b_{22} & =\frac{1}{4 I_{b}\left(\omega_{2}^{2}-4 \omega_{1}^{2}\right)}\left[2 \omega_{1}^{2} I_{b}\left(1-2 K I_{a}\right)+K\left(\omega_{1}^{2}\left(3-I_{a}^{2}\right)+2+K^{2}\right)-\alpha_{E} k \Delta\right] \\
c_{22} & =\frac{1}{4 I_{b} \omega_{2}^{2}}\left[K^{2}+2+\omega_{1}^{2}\left(1+I_{a}^{2}\right)-\alpha_{E} K \Delta\right] \\
\Phi_{22} & =a_{22} \cosh \left(\omega_{2} \tau\right)+\left(b_{22}+S_{22}\right) \cosh \left(2 \omega_{1} \tau\right)+\left(b_{22}+s_{22}\right) \\
\Delta & =2 K \gamma(2 K)(2 K \gamma(K)-1)+K \gamma(K)(K \gamma(K)-4)+3-3 K^{2} \\
S_{22} & =\frac{1}{8}(2 K \gamma(K)-1)
\end{aligned}
$$




$$
\begin{aligned}
P_{22}(\tau)= & \frac{\omega_{1}}{4}\left(1-2 K I_{a}\right), \quad D_{2}(\tau)=-\frac{1}{8}\left(1+\cosh \left(2 \omega_{1} \tau\right)\right), \\
F(\tau)= & -\frac{\tau}{8}\left[\omega_{1}^{2}\left(1-I_{a}^{2}\right)+\left(3-K^{2}\right)-\alpha_{E}\{\Delta+(1-2 K \gamma(2 K))\}\right] \\
& -\frac{1}{16 \omega_{1}} \sinh \left(2 \omega_{1} \tau\right)\left[\omega_{1}^{2}\left(3+I_{a}^{2}\right)+\left(3-K^{2}\right)-\alpha_{E}(\Delta+1)\right],
\end{aligned}
$$

and

$$
\omega_{2}^{2}=\frac{2 K}{I_{b}}\left(1-4 K^{2}\right)+\alpha_{E}(2 K \gamma(2 K)-1)
$$

with

$$
I_{b}=\frac{I_{0}(2 K)}{I_{1}(2 K)}, \quad \gamma(2 K)=\frac{K_{1}(2 K)}{k_{0}(2 K)} .
$$

Equation (34) reveals that the surface deformation of the capillary jet is not sinusoidal due to the presence of the axial dependent and the second harmonic terms whose growth rates are different from the fundamental mode. The nonlinearity also affects the cutoff wavenumber which separates the stable from the unstable disturbances. The application of the method of straining the coordinates sometimes may give erroneous results near the cutoff wavenumber $K_{\theta}$ [9]. In order to overcome this difficulty, we have used the method of multiple scales [7]. The dimensionless cutoff wavenumber turns out to be amplitude dependent and is given by (see Kent and Malik [10]):

$$
k_{c}=K_{\theta}+\eta_{0}^{2} k_{3}
$$

where $k_{c}=K_{\theta}$ is the cutoff wave number predicted by the linear theory and

$$
\begin{aligned}
k_{3}= & \frac{1}{4 K_{\theta}^{2}\left[2+\alpha_{E}\left(1-\gamma^{2}\left(K_{\theta}\right)\right)\right]} \\
& \times\left[\left(5+\frac{3 K_{\theta}^{4}}{2}-\frac{K_{\theta}^{2}}{2}\right)-2{ }_{2} S\left(1-K_{\theta}^{2}\right)\right. \\
& \quad+\alpha_{E}\left[\left\{2 K_{\theta}^{2} \gamma\left(K_{\theta}\right) \gamma\left(2 K_{\theta}\right)-2 K_{\theta}^{2}-4 K_{\theta} \gamma\left(K_{\theta}\right)\right\} S_{3}\right. \\
& \quad-\left(S_{2}-1\right)\left(K_{\theta}^{2}+2 K_{\alpha} \gamma\left(K_{\theta}\right)+1\right) \\
& \left.\left.\quad+3\left(K_{\theta} \gamma\left(K_{\theta}\right)\left(-K_{\theta} / 2+\gamma\left(K_{\theta}\right)+3\right)+\frac{3 K_{\theta}^{2}}{2}\right)\right]\right],
\end{aligned}
$$

with

$$
S_{3}=S_{2}+\left(K_{\theta} \gamma\left(K_{\theta}\right)-\frac{1}{2}\right)
$$

It may be noted that the results obtained by Nayfeh [9] for the same problem when the electricfield is absent can be deduced from equation (48) when $\alpha_{E}=0$ and $K_{0}=1$. 
5. Third order problem. The first and second order solutions of Secs. 3 and 4 can now be used to derive the solution to the third problem formulated below.

$$
\begin{aligned}
& L\left(\Omega_{3}\right)=2 k_{1} k_{3} K \frac{I_{0}(K r)}{I_{0}(K)} \sinh \left(\omega_{1} \tau\right) \cos (K \xi), \\
& L\left(\Phi_{3}\right)=2 k_{1} k_{3} K \frac{K_{0}(K r)}{K_{0}(K)} \cosh \left(\omega_{1} \tau\right) \cos (K \xi),
\end{aligned}
$$

with the boundary conditions at $r=1$ as

$$
\begin{gathered}
-\frac{\partial \eta_{3}}{\partial \tau}+\frac{\partial \Omega_{3}}{\partial r}=P_{31}(\tau) \cos (K \xi)+P_{33}(\tau) \cos (3 K \xi) \\
\Phi_{3}+\frac{\partial \Phi_{0}}{\partial r} \eta_{3}=R_{31}(\tau) \cos (K \xi)+R_{33}(\tau) \cos (3 K \xi) \\
-\frac{\partial \Omega_{3}}{\partial \tau}+\left(1+\frac{\partial^{2}}{\partial \xi^{2}}\right) \eta_{3}+\alpha_{E}\left[\left(\frac{\partial \Phi_{0}}{\partial r}\right)\left(\frac{\partial \Phi_{3}}{\partial r}\right)+\eta_{3}\left(\frac{\partial \Phi_{0}}{\partial r}\right)\left(\frac{\partial^{2} \Phi_{0}}{\partial r^{2}}\right)\right] \\
=Q_{31}(\tau) \cos (K \xi)+Q_{33}(\tau) \cos (3 K \xi),
\end{gathered}
$$

and the initial conditions

$$
\eta_{3}(\xi, 0)=0, \quad \partial \eta_{3}(\xi, 0) / \partial \tau=0
$$

where the expressions for $P, Q$ and $R$ are given in the Appendix B. Following an approach similar to that of Sec. 4 , we assume

$$
\eta_{3}(\xi, \tau)=B_{31}(\tau) \cos (K \xi)+B_{33}(\tau) \cos (3 K \xi) .
$$

The third order electric and velocity potentials now take the form

$$
\begin{gathered}
\Phi_{3}=\left[\left(B_{31}(\tau)+R_{31}(\tau)\right) \frac{K_{0}(K r)}{K_{0}(K)}-k_{1} k_{3} K r \frac{K_{1}(K r)}{K_{0}(K)}+k_{1} k_{3} K \gamma(k) \frac{K_{0}(K r)}{K_{0}(K)}\right] \\
\times \cos (K \xi)+\left[B_{33}(\tau)+R_{33}(\tau)\right] \frac{K_{0}(3 K r)}{K_{0}(3 K)} \cos (3 K \xi) \\
\Omega_{3}=\left[\left\{\frac{\partial B_{31}(\tau)}{\partial \tau}+P_{31}(\tau)-k_{1} k_{3} \omega_{1} K I_{a} \sinh \left(\omega_{1} \tau\right)\right\} \frac{I_{0}(K r)}{K I_{1}(K)}\right. \\
+\left\{\frac{\partial B_{33}(\tau)}{\partial \tau}+P_{33}(\tau)\right\} \frac{I_{0}(3 K r)}{3 K I_{1}(3 K)} \cos (3 K \xi) \\
\end{gathered}
$$

On substituting from equations (58) and (59) into equation (55), and equating the coefficients of $\cos (K \xi)$, we get the following differential equation for $B_{31}(\tau)$ : 


$$
\begin{aligned}
\frac{d^{2} B_{31}}{d \tau^{2}}(\tau)-\omega_{1}^{2} B_{31}( & \tau) \\
= & -\left[\mu_{1} p_{311}+\frac{K}{I_{a}}\left(q_{311}-\alpha_{E} K \gamma(K) R_{311}\right)\right] \cosh \left(\mu_{1} \tau\right) \\
& -\left[\mu_{2} p_{312}+\frac{K}{I_{a}}\left(q_{312}-\alpha_{E} K \gamma(K) R_{312}\right)\right] \cosh \left(\mu_{2} \tau\right) \\
& -\left[3 \omega_{1} p_{313}+\frac{K}{I_{a}}\left(q_{313}-\alpha_{E} K \gamma(K) R_{313}\right)\right] \cosh \left(3 \omega_{1} \tau\right) \\
& -\left[\omega_{1} p_{314}+\frac{K}{I_{a}}\left(q_{314}-\alpha_{E} K \gamma(K) R_{314}\right)-k_{1} k_{3} K\left\{\omega_{1}^{2}\left(I_{a}-\frac{1}{I_{a}}\right)\right.\right. \\
& \left.-\frac{\alpha_{E} K^{2}}{I_{a}}\left(1-\gamma^{2}(K)\right)\right\} \cosh \left(\omega_{1} \tau\right) .
\end{aligned}
$$

The constant $\nu_{3}$ appearing in $q_{314}$ in equation (60) is evaluated by requiring $\eta_{3}$ to be bounded for $K>K_{\theta}$. Towards that purpose, we set to zero the coefficient of the term involving $\cosh (\omega, \tau)$ in equation $(60)$ :

$$
\begin{aligned}
\nu_{3}= & \frac{1}{8} \gamma\left(1+K I_{a}\right)-\frac{\alpha}{8}\left(\frac{K}{I_{a}}+2\right)-\frac{\beta}{8}\left(1-2 K I_{b}+\frac{K}{I_{a}}\right) \\
& +\frac{1}{64}\left[8-5 K I_{a}+\frac{9 K}{I_{a}}\right]+\frac{K}{2} k_{1} k_{3}\left(I_{a}-\frac{1}{I_{a}}\right) \\
& +\frac{K \alpha_{E}}{4 I_{a}}\left[\left(A_{1}-A_{2}\right)\left(b_{M}+2 c_{M}\right)+(K \gamma(K)-1)\left(b_{M}+2 c_{M}\right)+6 K \gamma(2 K) b_{M}\right],
\end{aligned}
$$

where

$$
\begin{aligned}
& b_{M}=\frac{1}{4 I_{b} \omega_{2}^{2}}\left[2 I_{b}\left(1-2 K I_{a}\right)+K\left(3-I_{a}^{2}\right)\right], \\
& c_{M}=\frac{1}{4 I_{b} \omega_{2}^{2}}\left[\left(1+I_{a}^{2}\right)\right] .
\end{aligned}
$$

Similarly, we can obtain the differential equation for $B_{33}(\tau)$ by equating the coefficients of $\cos (3 K \xi)$ in equation (56). These differential equations furnish the following situations for $B_{31}(\tau)$ and $B_{33}(\tau)$ :

$$
\begin{aligned}
B_{31}(\tau)= & a_{31} \cosh \left(\omega_{1} \tau\right)+b_{31} \cosh \left(\mu_{1} \tau\right)+c_{31} \cosh \left(\mu_{2} \tau\right)+d_{31} \cosh \left(3 \omega_{1} \tau\right), \\
B_{33}(\tau)= & a_{33} \cosh \left(\omega_{3} \tau\right)+b_{33} \cosh \left(\mu_{1} \tau\right)+c_{33} \cosh \left(\mu_{2} \tau\right) \\
& +d_{33} \cosh \left(3 \omega_{1} \tau\right)+e_{33} \cosh \left(\omega_{1} \tau\right)
\end{aligned}
$$


where

$$
\begin{gathered}
a_{31}=-\left(b_{31}+c_{31}+d_{31}\right), \\
b_{31}=-\left[\mu_{1} p_{311}+\frac{K}{I_{a}}\left(q_{331}+\alpha_{E}\left(S_{331}-K \gamma(K) R_{311}\right)\right)\right] \frac{1}{\mu_{1}^{2}-\omega_{1}^{2}}, \\
c_{31}=-\left[\mu_{2} p_{312}+\frac{K}{I_{a}}\left(q_{312}+\alpha_{E}\left(S_{312}-K \gamma(K) R_{312}\right)\right)\right] \frac{1}{\mu_{2}^{2}-\omega_{1}^{2}}, \\
d_{31}=-\left[3 \omega_{1} p_{313}+\frac{K}{I_{a}}\left(q_{313}+\alpha_{E}\left(S_{313}-K \gamma(K) R_{313}\right)\right)\right] \frac{1}{8 \omega_{1}^{2}}, \\
a_{33}=-\left(b_{33}+c_{33}+d_{33}+e_{33}\right), \\
b_{33}=-\left[\mu_{1} p_{331}+3 K I_{c}\left(q_{331}+\alpha_{E}\left(S_{331}-3 K \gamma(K) R_{331}\right)\right)\right] \frac{1}{\mu_{1}^{2}-\omega_{3}^{2}}, \\
c_{33}=-\left[\mu_{2} p_{332}+3 K I_{c}\left(q_{332}+\alpha_{E}\left(S_{332}-3 K \gamma(K) R_{332}\right)\right)\right] \frac{1}{\mu_{2}^{2}-\omega_{3}^{2}}, \\
d_{33}=-\left[3 \omega_{1} p_{333}+3 K I_{c}\left(q_{333}+\alpha_{E}\left(S_{333}-3 K \gamma(K) R_{333}\right)\right)\right] \frac{1}{9 \omega_{1}^{2}-\omega_{3}^{2}}, \\
I_{33}=-\left[\omega_{1} p_{334}+3 K I_{c}\left(q_{334}+\alpha_{E}\left(S_{334}-3 K \gamma(K) R_{334}\right)\right)\right] \frac{1}{\omega_{1}^{2}-\omega_{3}^{2}}, \\
e_{3}^{2}(3 K) \\
I_{c}\left(\left(1-9 K^{2}\right)+\alpha_{E}(3 K \gamma(3 K)-1)\right], \quad \gamma(3 K)=\frac{K_{1}(3 K)}{K_{0}(3 K)},
\end{gathered}
$$

6. Numerical results and discussion. The distortion of the free surface is given by

$$
\begin{aligned}
\eta= & \eta_{0} \cos (K \xi) \cosh \left(\omega_{1} \tau\right) \\
& +\eta_{0}^{2}\left[B_{22}(\tau) \cos (2 K \xi)-\frac{1}{8}\left(\cosh \left(2 \omega_{1} \tau\right)+1\right)\right] \\
& +\eta_{0}^{3}\left[B_{31}(\tau) \cos (K \xi)+B_{33}(\tau) \cos (3 K \xi)\right],
\end{aligned}
$$

where

$$
K=\frac{k}{k_{c}}, \quad \xi=k_{c} Z, \quad \tau=\nu t \quad \text { with } k_{c}=k_{1}+\eta_{0}^{2} k_{3} .
$$

In addition to the fundamental mode, the presence of various harmonics in equation (76) is the result of the energy transfer from the fundamental mode to the modes of higher orders. To obtain the time of breakup of the jet, the time $\tau$ appearing in the equation (76) is allowed to increase such that the deepest trough of the wave coincides with the axis of the capillary jet. Physically, this criterion makes sense. It also explains experimental observations for the hydrodynamic jet. With this criterion for the breakup, a numerical 
search for the breakup points and the breakup times was carried out simultaneously by increasing $\tau$ and assigning values to $\xi$ in the equation (76) until we get $r=0$ i.e., $\eta(\xi, \tau)=-1$. Once critical $\tau$ has been determined, we can then use equation (76) to furnish a profile of $\eta$ versus $\xi$ for different values of $\alpha_{E}$. We observe that the jet breakup time first decreases and then increases with the increase of the electric field parameter $\alpha_{E}$.

The volumes of the liquid contained by the primary wave (main drop) and the secondary wave (satellite) are calculated analytically. Assuming that the resultant drops as well as satellites would be spherical in shape, their volumes are converted into equivalent radii $R_{M}$ and $R_{S}$, respectively. These radii have been normalized with respect to the radius of the jet and profiled in Fig. IV. It is found that the initial amplitude of $\eta_{0}$ (ranging from $10^{-1}$ to $10^{-4}$ ) does not affect qualitatively the results summarized in Fig. IV. It should be remarked here that Rutland and Jameson [3] and Lafrance [8] show the agreement between experiment and theory for the unelectrified jet to be reasonably good when the wavenumber $K$ lies between 0.3 and 0.5 . In this range, the main and satellite drops are of the same size, and there is no satellite production beyond the point of maximum instability. We recover these results by setting $\alpha_{E}=0$ as indicated in Figure IV. However, it is interesting to note that the satellite are always present for all values of wavenumbers when an external electric field is applied to the jet. This, however, has not yet been confirmed or denied so far by experiments.

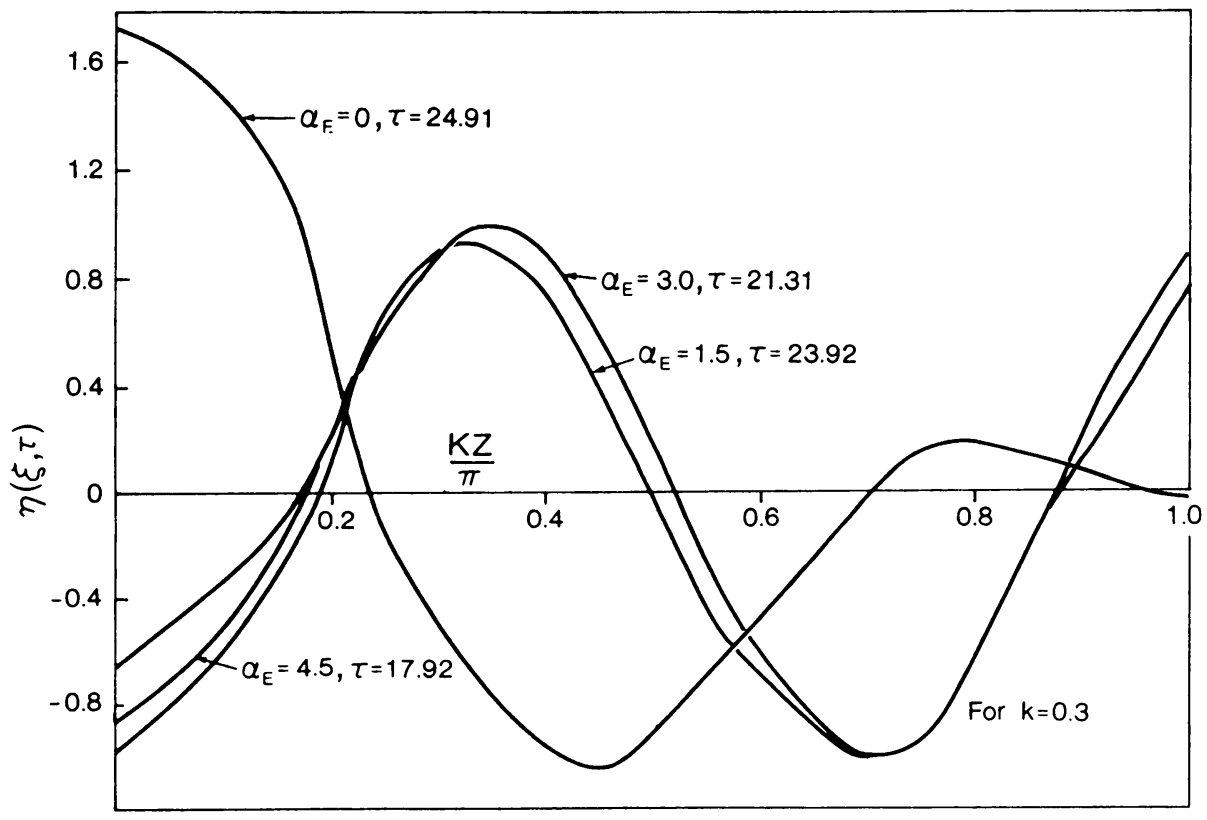

FIG. I. Wave profiles at the breakup for the dimensionless wavenumber $k=0.3$ and $\alpha_{E}=0,1.5,3.0,4.5\left(\eta_{0}=0.01\right)$.

The behaviour of the nonlinear surface profiles of the jet are sketched in Fig. I to III. The Fig. I and II show that the introduction of the electric field induces the surface profile to change drastically in that the surface elevation $\eta$ starts from negative values and then 
keeps on oscillating. This trend continues until $K$ reaches the value 0.678 . For $K=0.9$, Fig. III illustrates that the trend is reversed. The variation of the radii of the main drops and satellites as a function of the wavenumber and the applied electric field is demonstrated in Fig. IV. For $\alpha_{E}=0$, there are no satellites beyond the point of maximum instability. However, as the values of $\alpha_{E}$ increase, the satellite radii go on decreasing up to the point of maximum instability and then start increasing till the wavenumber $K$ reaches the value 0.8 . For $K$ larger than 0.8 , the satellite radii decrease again but very slowly.

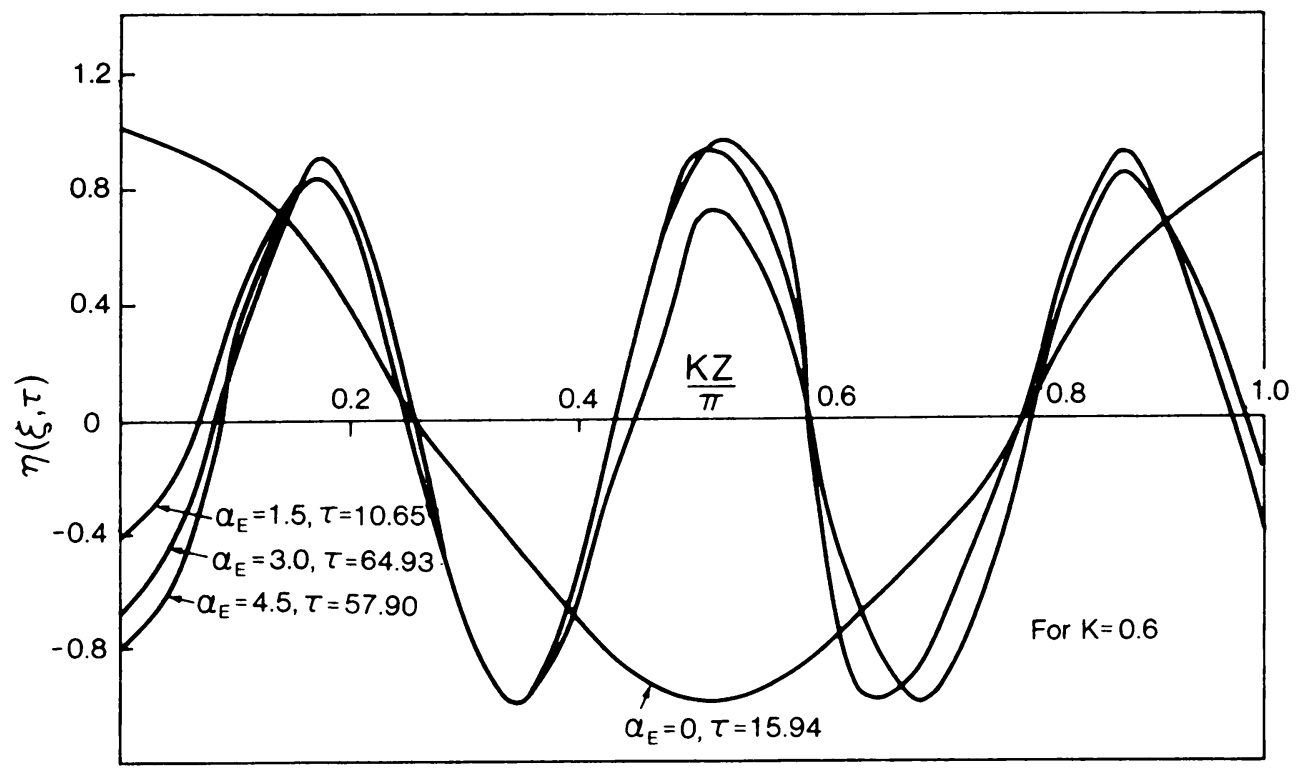

FIG. II. Wave profiles at the breakup for the dimensionless wavenumber $k=0.6$ and $\alpha_{E}=0,1.5,3.0,4.5\left(\eta_{0}=0.01\right)$.

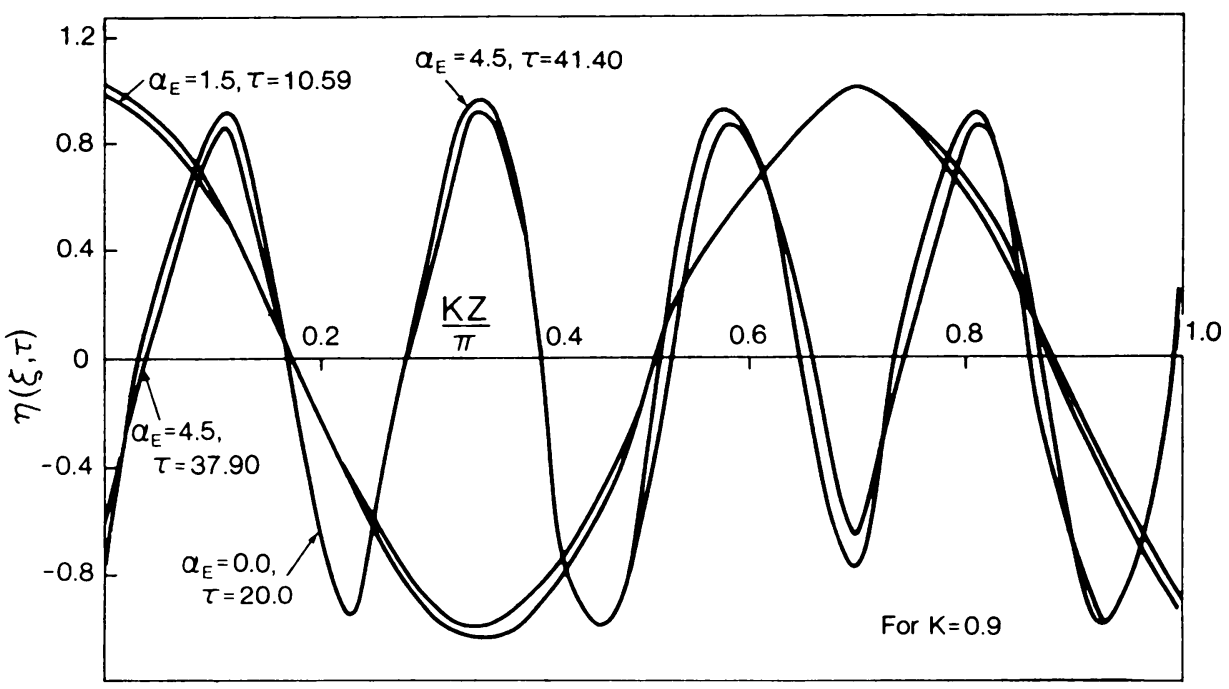

FIG. III. Wave profiles at the breakup for the dimensionless wavenumber $k=0.9$ and $\alpha_{E}=0,1.5,3.0,4.5\left(\eta_{0}=0.01\right)$. 


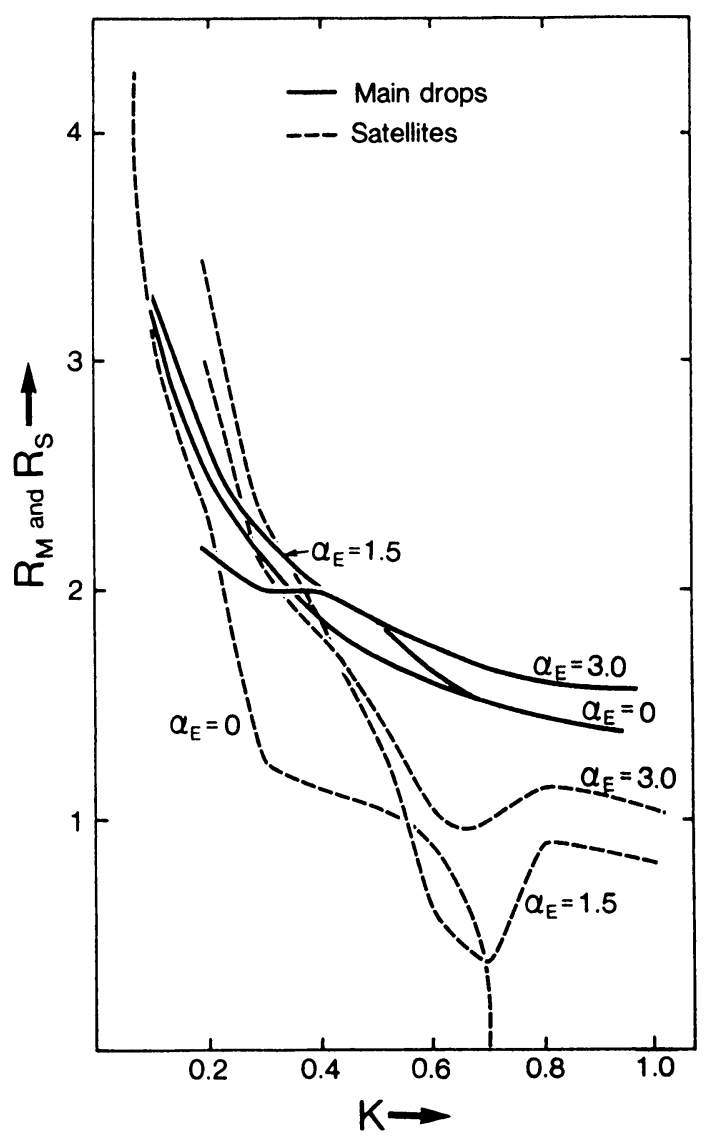

FIG. IV. Comparison of the predicted main drops and satellite sizes from the breakup of electrohydrodynamic jet.

Appendix A. The equation of motion of the conducting electrohydrodynamics fluid is

$$
\rho \frac{\partial V_{i}}{\partial t}+V_{j} \frac{\partial}{\partial x_{j}} V_{i}=-\frac{\partial p}{\partial x_{i}}+\frac{\partial}{\partial x_{j}}\left(E_{i j}\right)
$$

where $E_{i j}$ denotes the Maxwell stress given by

$$
E_{i j}=\frac{1}{4 \pi}\left(E_{i} E_{j}-\frac{1}{2} E_{k} E_{k} \delta_{i j}\right) .
$$

In order to obtain the condition that the normal stress be continuous at the deformed boundary, we integrate (A1) across the boundary which yields

$$
n_{i}[p]-n_{j}\left[E_{i j}\right]=0,
$$

where the square bold bracket indicates the difference in the values of the variable evaluated in two regions across the surface. For a capillary jet subjected to an external electric field, equation (A3) takes the form

$$
-p+T\left(\frac{1}{R_{1}}+\frac{1}{R_{2}}\right)-\frac{1}{8 \pi}\left(\frac{\partial \Phi}{\partial \hat{n}}\right)^{2}=0,
$$

where $R_{1}$ and $R_{2}$ are the principal radii of curvature. The dynamic pressure $p$ in (A4) can 
be evaluated with the use of the Bernoulli's equation. We obtain

$$
p=f(t)-\frac{1}{2} \rho\left[\left(\frac{\partial \Omega}{\partial r}\right)^{2}+\left(\frac{\partial \Omega}{\partial Z}\right)^{2}\right]-\rho\left(\frac{\partial \Omega}{\partial t}\right),
$$

where $f(t)$ stands for the constant of integration with respect to the space variables.

The expression for the second term in (A4) can be derived from solid geometry. If $\nu_{1}, \nu_{2}, \nu_{3}$ are the direction cosines of the normals at $(r, \theta, Z)$ to the surface of the family

$$
f(r, \theta, Z)=r-\eta(Z, t)-1=0,
$$

then

$$
\left(\nu_{1}, \nu_{2}, \nu_{3}\right)=\left(\frac{\partial f}{\partial r}, \frac{1}{r} \frac{\partial f}{\partial \theta}, \frac{\partial f}{\partial z}\right)\left[\left(\frac{\partial f}{\partial r}\right)^{2}+\frac{1}{r^{2}}\left(\frac{\partial f}{\partial \theta}\right)^{2}+\left(\frac{\partial f}{\partial z}\right)^{2}\right]^{-1 / 2},
$$

and following Lamb [11, p. 475],

$$
\begin{aligned}
\frac{1}{R_{1}}+\frac{1}{R_{2}} & =\frac{1}{r} \frac{\partial}{\partial r}\left(r \nu_{1}\right)+\frac{1}{r} \frac{\partial}{\partial \theta}\left(\nu_{2}\right)+\frac{\partial \nu_{3}}{\partial Z} \\
& =(1+\eta)^{-1}\left(1+\left(\frac{\partial \eta}{\partial Z}\right)^{2}\right)^{-1 / 2}-\frac{\partial^{2} \eta}{\partial Z^{2}}\left(1+\left(\frac{\partial \eta}{\partial Z}\right)^{2}\right)^{-3 / 2} .
\end{aligned}
$$

From the results (A4)-(A6), we get

$$
\begin{aligned}
& \frac{1}{8 \pi}\left(\frac{\partial \Phi}{\partial \hat{n}}\right)^{2}-\rho\left(\frac{\partial \Omega}{\partial t}\right)-\frac{\rho}{2}\left[\left(\frac{\partial \Omega}{\partial r}\right)^{2}+\left(\frac{\partial \Omega}{\partial z}\right)^{2}\right] \\
& +T\left(1+\left(\frac{\partial \eta}{\partial Z}\right)^{2}\right)^{-1 / 2}\left\{\frac{\partial^{2} \eta}{\partial Z^{2}}\left(1+\left(\frac{\partial \eta}{\partial Z}\right)^{2}\right)^{-1}+(1+\eta)^{-1}\right\}+T=0,
\end{aligned}
$$

at $r=1+\eta(Z, t)$.

For an unelectrified jet, the above equation (A7) reduces to that obtained by Yuen [4].

Appendix B. The coefficients $P$ and $Q$ are given in Yuen's paper [4] while $R$ and $S$ are

$$
\begin{gathered}
R_{311}=R_{312}=\left(a_{22} / 4\right)[K \gamma(K)+2 K \gamma(K)-1], \\
R_{313}=\frac{1}{4}\left[b_{22}(K \gamma(K)-1)+2 K \gamma(2 K)\left(b_{22}+S_{22}\right)-0.75(K \gamma(K)-1)\right. \\
\quad-0.25\{1.5 K(K+\gamma(K))-1\}], \\
R_{314}=\frac{1}{4}\left[\left(b_{22}+2 c_{22}\right)(K \gamma(K)-1)+\left(b_{22}+S_{22}\right) 6 K \gamma(2 K)\right. \\
\quad-0.25(K \gamma(K)-1)-0.75\{1.5(K(K+\gamma(K))+1)\}], \\
\left.R_{331}=R_{311}=R_{332}, \quad-0.25\left\{0.5 K(K+\gamma(K))-\frac{1}{3}\right\}\right], \\
R_{333}=\frac{1}{4}\left[b_{22}(K \gamma(K)-1)+2 K \gamma(2 K)\left(b_{22}+S_{22}\right)\right. \\
R_{334}=\frac{1}{4}\left[\left(b_{22}+2 c_{22}\right)(K \gamma(K)-1)+6 K \gamma(2 K)\left(b_{22}+S_{22}\right)\right. \\
\left.-\frac{3}{4}\left\{0.5 K(K+\gamma(K))-\frac{1}{3}\right\}\right],
\end{gathered}
$$




$$
\begin{aligned}
& S_{311}=S_{312}=-\left(a_{22} / 4\right)\left[A_{1}-A_{2}+2 K^{2}\right] \text {, } \\
& S_{313}=-\frac{1}{4}\left[\left(A_{1}-A_{2}\right) b_{22}+A_{1} S_{22}+\frac{1}{4}\left(K^{2}+2 K \gamma(K)+1\right)+\frac{3}{4} A_{3}\right. \\
& \left.+2 K^{2}\left(b_{22}+S_{22}\right)-\frac{1}{4} K^{3} \gamma(K)\right], \\
& S_{314}=-\frac{1}{4}\left[\left(A_{1}-A_{2}\right)\left(b_{22}+2 c_{22}\right)+3 A_{1} S_{22}+\frac{9}{4} A_{3}\right. \\
& \left.+\left(b_{22}+2 c_{22}+S_{22}\right) 2 K^{2}-\frac{3}{4} K^{3} \gamma(K)+\frac{3}{4}\left(K^{2}+2 K \gamma(K)+1\right)\right], \\
& S_{331}=S_{332}=-\left(a_{22} / 4\right)\left[A_{1}-A_{2}-2 K^{2}\right] \text {, } \\
& S_{333}=-\frac{1}{4}\left[\left(A_{1}-A_{2}\right) b_{22}+A_{1} S_{22}+\frac{A_{3}}{4}+3 K^{2}\left(b_{22}+S_{22}\right)+\left(K^{3} / 4\right) \gamma(K)\right] \text {, } \\
& S_{334}=-\frac{1}{4}\left[\left(A_{1}-A_{2}\right) b_{22}+S_{22} A_{1}+A_{3} / 4+\frac{3}{4} K^{3} \gamma(K)-2 K^{2}\left(b_{22}+S_{22}\right)\right],
\end{aligned}
$$

where

$$
\begin{aligned}
& A_{1}=2 K^{2} \gamma(K) \gamma(2 K)-4 K^{2}(1+\gamma(K) / 2 K)-2 K \gamma(2 K), \\
& A_{2}=K^{2}+2 K \gamma(K)+1 \\
& A_{3}=K \gamma(K)\{-K(K / 2+\gamma(K)+3)\}+\frac{3}{2} K^{2}-2
\end{aligned}
$$

\section{REFERENCES}

[1] J. W. S. Rayleigh, Theory of sound, Vol. 2, Macmillin, London, 2nd ed., 1896; reprinted Dover, New York, 1945, 1947

[2] R. J. Donelly and W. Glaberson, Experiments on the capillary instability of a liquid jet, Proc. Roy. Soc. London A 290, 547 (1966)

[3] D. F. Rutland and G. J. Jameson, A nonlinear effect in the capillary instability of liquid jets, J. Fluid Mech. 46, 267 (1971)

[4] M. C. Yuen, Nonlinear capillary instability of a liquid jet, J. Fluid Mech. 33, 151 (1968)

[5] J. R. Melcher, Field coupled surface waves, M.I.T. Press, Cambridge, Mass., 1963

[6] S. K. Malik, M. Singh and W. Welsh, Nonlinear breakup of a self-gravitating column, J. Math. Annal. Appl., 89, 370 (1982)

[7] A. H. Nayfeh, Perturbation methods, Wiley, New York, 1973

[8] P. Lafrance, Nonlinear breakup of a laminar liquid jet, Phys. Fluids, 18, 428 (1975)

[9] A. H. Nayfeh, Nonlinear instability of a liquid jet, Phys. Fluids, 13, 841 (1970)

[10] R. Kant and S. K. Malik, Nonlinear electrohydrodynamic instability of a jet (to appear)

[11] H. Lamb, Hydrodynamics, sixth edition, Cambridge, 1975 